\title{
USE OF CARE PATHS TO IMPROVE PATIENT MANAGEMENT
}

\author{
Suzann K. Campbell
}

Suzann K. Campbell, PT, PhD, FAPTA, is Professor Emerita, University of Illinois at Chicago, Department of Physical Therapy, and Manager, Infant Motor Performance Scales, LLC, Chicago, IL, USA. Address correspondence to the author at 1301 W. Madison Street \#526, Chicago, IL 60607, USA, or via email to skc@uic.edu.

The purpose of this special issue of Physical \& Occupational Therapy in Pediatrics is to present an evidence-based system to guide the physical therapy management of patients in the Neonatal Intensive Care Unit (NICU). Two systematic guides to patient management will be presented. The first is a care path intended primarily for use by physical therapists, and the second is a care path for families of infants being cared for in the NICU. In this article background information on the concept of using care paths for clinical management is presented, followed by a general description of the two care paths for use in the NICU. Subsequent articles describe physical therapy practice in the context of the NICU team approach to care of medically fragile infants with more detail on use of the two care paths and the evidence supporting their contents.

The Care Path Concept

The care path (variously called critical path, clinical path, clinical pathway, clinical protocol, care map, and care track) is a recommended diagnostic and treatment regimen that includes time- or outcome-dependent functions used to standardize the patient care process throughout a course of treatment (Renholm, Leino-Kilip, \& Suominen, 2002). A care path details the essential steps in patient care while describing the expected progress of the patient. It is a guide to the standard of care and expected outcomes within a specified period of time for a 
particular group of patients (Schwoebel \& Helfrich, 1999). Use of a care path promotes coordination of care and the achievement of specific patient outcomes within a specified period of time. The typical care path should be appropriate for the care of about $80 \%$ of patients in the service setting; variation from the path must be allowed for in especially complicated cases and having a care path helps to assure early recognition of needed variances from typical practice expectations. Care paths are not cookbooks, but rather they provide guidelines for best practice, specify expected outcomes within defined time periods, and provide assistance in prioritizing care and avoiding accidental omission of critical services. Evaluation of the need for frequent variances from the path and identification of why they occur can lead to improvements in services provided.

Clinical care or diagnostic pathways have been developed in a variety of health care fields such as orthopedics, neurology, pulmonary disease, and pediatrics, including neonatal nursing care management (Gretebeck, Shaffer, \& Bishop-Kurylo, 1998; Krebs, 1998; Jones \& Smyth, 1999; Renholm, et al., 2002; Schwoebel \& Helfrich, 1999). The critical path method of project management was first introduced in the petrochemical industry in the 1950s and was first used in health care in the mid-1980s (Schwoebel \& Helfrich, 1999). In today's competitive health care system clinical care paths are considered one of the appropriate tools to use to improve quality of care while controlling costs.

Although research on the outcomes of using care paths to manage patients is relatively sparse given the length of time over which they have been used, Renholm and colleagues (2002) reviewed evidence indicating that their utilization in health care can improve quality of care, patient satisfaction and education, as well as continuity of care and communication of 
information, and reduce costs primarily through shortening the length of hospital stay and avoiding duplication of diagnostic testing.

The goal of developing a care path typically requires interdisciplinary involvement in order to improve communication among the members of the health care team taking care of a specific group of patients (Renholm, et al., 2002), and several examples exist in the neonatal care literature (Elizondo, 1995; Jones \& Smyth, 1999; Schwoebel \& Helfrich, 1999). The development of the existing pathways, however, did not typically include input from physical therapists so the content primarily reflects the concerns of nurses and, to some extent, physicians. For example, Schwoebel and Helfrich (1999; Jones \& Smyth, 1999) developed a pathway system for the NICU called NeoMaps (neonatal multidisciplinary action plans) based on input from a team including neonatologists, pharmacists, a social worker, laboratory representatives, a variety of nurse specialists, and an infant developmental specialist (professional discipline unspecified).

NeoMaps were developed for infants in seven different age groups from less than 26 weeks postmenstrual age (PMA) through 44 weeks PMA (Jones \& Smyth, 1999). The care paths included expected aspects of care and also variance paths to record information on patients who needed to be cared for off the pathway because of complications or other circumstances that did not allow them to follow the standard plan of care. Aspects of care included in the standard map were respiratory, cardiac, and nutritional care; thermal regulation, neurodevelopment (limited to oral motor progression and individualized developmental support for environmental protection); medications, consults, and diagnostic studies; and family teaching and discharge planning. The process used to design NeoMaps is typical of how such guides to care are developed. The members of the multidisciplinary team each identified critical aspects of their care from review of medical records and documented when during the inpatient stay these services were typically 
provided (Schwoebel \& Helfrich, 1999). The team met to consider areas of overlap and then each discipline developed a profile of what future practice should be. After a first draft of the care path was designed, members of the developmental team consulted with their disciplinary colleagues to assess agreement with the plan. Consensus-building continued until all disciplinary representatives approved the pathway, a process which took about 5 months. The pathway was implemented; after 2 months, areas for improvement were identified and addressed so that the pathway clearly identified infant needs, goals and desired outcomes with tasks and activities clearly defined. Initial implementation problems with the pathway included limited use by those other than nurses, concerns regarding the volume of documentation and paperwork required (particularly in areas that were not being used clinically), and inadequate guidance with regard to discharge planning throughout the infant's stay. Eventually the revised care path was incorporated into the regular medical record to reduce the documentation burden and assure more coordinated care. Considerable redundancy was identified in the maps for different PMAs; as a result age-specific maps were dropped in favor of one map for all infants, but two discharge pathways were used, one for infants born prematurely and one for infants born at term.

Jones and Smyth (1999) conducted a study of the outcomes of using the NeoMaps for two specific diagnostic-related groups as compared with their conventionally managed care in the previous time period and found a number of non-statistically significant improvements, such as a reduction of time to total nipple feeding. Infant motor development was not assessed as it was not addressed in the NeoMap guidelines. Overall no significant effects of instituting managed care were found, but evaluation of the results was hindered by an increase in complications such as pulmonary conditions and extreme prematurity over the two time periods. The fact that length of stay and costs did not change from one time period to the next may have 
actually been a result of the institution of pathway-guided care being effective in the face of having to manage a more complicated patient population.

Other examples of care paths identified in the NICU literature are more limited in scope. One such is a feeding care map for the extremely low birthweight infant (Dougherty \& Luther, 2008). Groups of tasks are organized by days of life and corrected age and include such activities as skin-to-skin care (kangaroo care), breast pumping, nutritive suck development, and positioning and other supports for feeding.

Other examples include care paths to guide family-focused developmental care activities to promote infant behavioral organization. A neonatal nurse practitioner designed a clinical pathway for parent education in the NICU based on review of the literature on individualized developmental care and her clinical experience (Krebs, 1998). Elements of the care path included parents being able to recognize and respond to infant cues for social engagement, demonstration of handling and positioning techniques, and developing independence in all aspects of the infant's caregiving prior to discharge. The path progressed from day of admission by 2 -week increments through 36 weeks PMA or discharge, but did not specify any changes in expectations across infant age. A second example of family-oriented care plans was developed by a neonatologist, developmental specialist, and nurse (Gretebeck, et al., 1998). A notable result of the introduction of this care path was that the provision of practical sources of information to families increased from $4 \%$ to $100 \%$ of families.

Physical Therapy Care Paths

The next section provides a description of the process by which the two care paths to be presented in this special issue were developed. The group came together after two of our members had independently developed care paths to guide their physical therapy practice in the 
NICU, one developed by Eilish Byrne for the therapist caring for inpatients, and the other developed by Lou Ann Goldstein to guide and educate parents about expectations for them and their baby from the time of birth through to discharge from the NICU.

The Infant Care Path for Physical Therapy in the Neonatal Intensive Care Unit (NICU Care Path) was created by Eilish Byrne, Lucile Packard Children's Hospital in California, to help guide the care provided by physical therapists in a NICU setting. After providing direct interventions and conducting research in this setting for many years, she began to think that an evidenced-based physical therapy care path could benefit practice. She began to discuss the idea with other neonatal physical therapists to obtain their opinion regarding the matter, and over time it became clear that the development of a formal care path would be of value. Byrne then drafted the first version of what would become the NICU Care Path and shared it with June Bridgeford Garber in Georgia who expanded the oral-motor portion of the care path. After that initial meeting four years ago, they agreed to find a team to assist with the project. As a result Suzann Campbell and Lou Ann Goldstein from Illinois joined the team to further develop the care path. Together this group of highly experienced professionals subsequently wrote evidenced-based corresponding articles to further explain the content of the NICU Care Path and provide support for the role of physical therapy in the NICU. It is this work that is being presented in this special issue of Physical \& Occupational Therapy in Pediatrics.

The second care path presented in this special issue is that for use by parents developed by Lou Ann Goldstein, Comer Children's Hospital at the University of Chicago. The main goal in developing the NICU Discharge Path was to ensure that all parents were provided with the essential baby care information in order to prepare the parents for their infant's discharge from the NICU. The process to develop the most essential categories was not an easy task. An 
interdisciplinary team consisting of a neonatologist, two nurse case managers, a social worker, a speech therapist, and a physical therapist provided input for design of the tool. Together, the team identified the infant care categories to be discussed with the parents as well as the appropriate time frames to introduce and reinforce the information. Parents would receive the most important information at the most important time in the infant's care. The design of the tool was developed in order to increase the parent's participation in their infant's care during the NICU hospitalization and to ensure their level of knowledge and competence needed when their infant was ready to go home. The order of the information was established so that certain educational areas could be introduced, reinforced, and expanded upon over the course of the infant's stay in the NICU to decrease the feeling of being overwhelmed with information as well as reinforcing more complex information or ensuring the parents' competency in other areas. Parents of complex infants who might need more specialized care would then have more time to develop the skills and competence for their infant's care, and they could also seek feedback from the health care team if questions developed. The design of the NICU Discharge Path with short phrases and pictures was developed to invite parents to participate in the discharge process and to be more accountable for their infant's care. The parents could easily read, understand, and anticipate each step of their infant's care and goals to achieve in order for their infant to be discharged home successfully. Preparing the parents for their infant's discharge from the NICU is equally as important as preparing the infant for discharge.

As the group of experienced neonatal physical therapists came together, input from an occupational therapy colleague was thought to be valuable, and Vanessa Barbosa from Illinois was added to the team. As we reviewed the contents of the care path and considered the degree of overlap among skills and services provided by the variety of developmental specialists who 
work in the NICU, the group engaged in active debate about whether it should be considered to be a physical therapy care path or a developmental therapy care path that could include occupational therapy and speech and language therapy as well as developmental specialists from nursing or psychology. Because role delineation is a frequent topic of discussion in special interest groups related to NICU practice, we have learned that it is common to employ both physical therapists and occupational therapists in NICUs in a way that assumes no difference between the professions (e.g., accepting referrals for services by alternating which professional accepts the patient). Although there is certainly significant overlap, each profession also has unique theoretical frameworks for practice and areas of expertise (see article by Barbosa). For example, in a student project in one of my graduate classes on NICU practice, an occupational therapist presented results of a developmental assessment done on an infant in the NICU to a group of physical therapists and occupational therapists. Although they were reviewing the same patient data, it was clear that members of each professional group saw the test results differently based on their theoretical perspective. Occupational therapists viewed the data in light of their emphasis on sensory processing and motor planning, while physical therapists focused more on postural control, quality of movement, and motor milestone achievement. As a result intervention plans derived from the assessment information differed by professional group. Based on this reflection on practice, we decided to include the article by Barbosa on Team Work in the NICU as the introduction to our work in acknowledgment of the unique as well as overlapping roles of the members of the NICU health care team.

We also believe that physical therapy practice involves more than just developmental care, and therefore ultimately decided that our care path would be specific to physical therapy. We agreed, however, that we would provide ample recognition of the fact that numerous tasks 
and activities included in the care path are ably offered by other developmental specialists and that who does what in a given unit is often a function of the training and experience of the individual professional in particular aspects of care rather than discipline-specific. Because the development of care paths is generally assumed to have been done by an interdisciplinary group, we do believe that it would be entirely appropriate for a team in any NICU to use our physical therapy care path as a basis for design of a developmental care path for their own unit that takes advantage of the unique experiences and educational background of the members of their particular team and service organization. Having a defined care path unique to physical therapy, however, provides a means of communicating to the NICU care team the breadth of physical therapist competencies for practice in this unique setting in an organized and brief document. The Infant Care Path for Physical Therapy in the Neonatal Intensive Care Unit (NICU Care Path) The competencies required by physical therapists working in the NICU environment are described by Sweeney and colleagues (Sweeney, Heriza, \& Blanchard, 2009) along with a clinical decision making algorithm for neonatal physical therapy that provides a guide to examination, evaluation of findings, intervention, and re-examination of the infant in the NICU. A second article (Sweeney, Heriza, Blanchard, \& Dusing, 2010) presents theoretical frameworks for practice and evidence-based practice guidelines. The purpose of our care path for physical therapy and developmental care in the NICU is to provide an adjunct to these competency and practice guidelines in order to simplify documentation and communication, facilitate adherence to evidence-based practice guidelines, prioritize care and prevent omissions of recommended tasks and activities. In addition, items on the NICU Care Path are arranged in a time-ordered, i.e., developmental, sequence, from infant admission to the NICU to discharge to home. The Infant Care Path for Physical Therapy in the Neonatal Intensive Care Unit (Fig. 2.1) is organized 
into three sections: Physical Therapy Observation and Assessment, Intervention (including Family Support and Education), and Team Collaboration. In each section a series of tasks and activities are listed along with recommendations for PMAs at which each is appropriate. When the boxes under specific PMAs are shaded, this indicates that the task or activity is not generally appropriate at that age. Open boxes typically show a range of PMAs for performing the task or activity and the therapist documenting services using the care path will record when the task was performed within the general range of age for which it was appropriate. As with any care path, the intent is to cover tasks and activities in an age-linked sequence which is appropriate for the majority of infants, but those with special complications may need variances from the recommended PMAs for performing an activity, and all variances from the path should be documented.

Physical Therapy Observation and Assessment includes sections for tasks involving 1) observation only, 2) limited hands-on assessment, 3) full hands-on assessment, and 4) sensory assessment. The article by Byrne and Campbell on Physical Therapy Observation and Assessment describes the evidence supporting inclusion of various types of examinations included in the care path. Like the guidelines for neonatal physical therapy (Sweeney, et al., 2009), we do not prescribe specific actions but rather present information on available options and the research evidence supporting use of these options. The physical therapist using the care path selects the most appropriate option for each infant, thus individualizing care. As a result the NICU Care Path is not a cookbook but a helpful guide to practice that encourages holistic services for infants and systematic documentation of the tasks and activities appropriate to the physical therapist's practice in the NICU. 
In similar fashion the Intervention section of the care path provides options for treatment under the headings of 1) facilitating calm state and motor organization, 2) positioning and handling, 3) movement therapy, 4) oral-motor organization and feeding, and 5) family support and education. The Family Support and Education section of the care path includes a variety of interventions supported by evidence in the literature, including activities such as helping families to interpret infant behavior and cues, skin-to-skin care, feeding activities, and facilitating motor development. Although practice in the NICU is always performed within the context of the care team, including the family, a special section on Team Collaboration in the care path reminds the physical therapist that developmental programming, positioning and developmental supports, anticipatory guidance for family members, and assessing the need for a variety of consults should be done in concert with the other members of the infant's health care team. Articles by Byrne and Garber in the special issue elaborate on the theory and evidence in the literature for these areas of emphasis in neonatal physical therapy practice. The NICU Discharge Path: Preparing for Your Baby to Come Home

The second care path presented in this special issue is one for use by parents in collaboration with their infant's caregivers (Fig. 2.2). The NICU Discharge Path: Preparing for Your Baby to Come Home is titled to encourage the family to engage thoughtfully in gaining the skills and knowledge that will lead to a successful homecoming for their baby, or as expressed by Dougherty and Luther (2008), to assist parents in navigating the course of a NICU stay. The categories of information included in the NICU Discharge Path include Nutrition, Baby Care, Medications and Equipment, Your Health Care Team, Tests, Education, and Discharge Planning. The time-sequenced tasks and activities for parents to engage in are presented across 5 epochs: At Admission, When Your Baby Weighs 1500-1600 g, When Your Baby Weighs 1600-1800 g, 
Two Weeks Before Discharge, and One Week Before Discharge. The purpose of this care path is to provide concrete guidance for families, parceled into discrete sections that can be accomplished one at a time while also providing anticipatory guidance that helps the family to look ahead to the next steps in the whole process of care during a NICU experience. The family can record their accomplishments, be reminded to ask about needed information if it is not offered, and be aware that the often chaotic-appearing environment is actually a carefully planned holistic service focused on achieving the best possible outcomes for them and their infant. We try to be as inclusive as possible while allowing for variances in tasks and activities by including the statement that "All activities may not apply to every baby." The article by Goldstein on Family Support and Education describes use of the NICU Discharge Path in more detail.

The importance of providing parents with materials to guide their preparation for the infant's discharge throughout the course of the NICU stay is well documented in the literature. As previously noted Schwoebel and Helfrich (1999) realized in the early development of the NeoMaps that discharge planning was not adequately addressed across the course of the infant's stay in the NICU and revised the care paths accordingly. Mills and colleagues (Mills, Sims, \& Jacob, 2006) similarly developed a system to improve the discharge process that involved identification of transition points spanning the entire hospitalization which was implemented in 6 hospitals in the Vermont Oxford Discharge Planning Collaborative (fancifully and aptly named the No Place Like Home Group). Interestingly, each hospital implemented the system differently, emphasizing that flexibility in accomplishing practice improvement goals is available to match responsive activities to institutional culture and standards. 
In addition to the goal of planning for discharge across the hospital stay the Discharge Planning Collaborative hoped to avoid the last day rush which often resulted in omission of important activities (Mills, et al., 2006). A major part of their redesigned process was the provision of caregiver educational tools. An important finding of their evaluation was that parents were overwhelmed by being given too much information at admission. Consequently information was subdivided into packages for presentation to families either in the early phase of orientation to the NICU or when preparing families to care for their infant at home. Evaluation of outcomes showed increased accomplishment of transition points tasks and improved staff satisfaction. As part of the evaluation process an Internet-based parent satisfaction survey was identified and successfully implemented. Centers are beginning to use the data from these surveys to change practice as indicated. Use of this survey is recommended as one way to evaluate the utility of the NICU Discharge Path presented in this special issue. The survey can be accessed at www.howsyourbaby.com .

Further development of the NICU Care Path and the NICU Discharge Path depends on research on the outcomes of their use, and it is our hope that one of the results of this publication will be more study and revision of the pathways as new evidence on practice emerges. Some of the questions that need to be addressed include:

1. Are all elements of the care plan for a given infant implemented during the time period specified in the path? If not, what are the barriers (and possible solutions) to implementation of the recommended activities and tasks?

2. Is the care path appropriate for about $80 \%$ of patients cared for in the unit? If not, what changes are needed? Are variances from the care path common in any groups of patients for 
whom special pathways need to be developed because their care is more complex than that of most patients in the unit?

3. Is the time required for documentation or coordination of care reduced by use of the care path?

4. Are costs of care reduced by use of the care path? What is the effect on time to discharge, frequency of duplication of services, or reduction of omissions in services provided?

5. What are the effects of care path use on infant development and on parent knowledge, satisfaction, and readiness for discharge?

In summary, this article provided information on the purposes and uses of clinical care paths, examples of care paths designed primarily by nurses for the NICU, research on outcomes of using this type of structured documentation and practice guidelines, and a general description of two care paths for physical therapy and developmental care in the NICU developed by our team of physical therapists and an occupational therapist. Further research to validate the usefulness of the care paths is recommended.

\section{REFERENCES}

Antevil, J.L., Rivera, L., Langenberg, B.J., Hahm, G., Favata, M.A., \& Brown C.V.R. (2006)

Computed tomography-based clinical diagnostic pathway for acute appendicitis:

Prospective validation. J Am Coll Surg, 203, 849-856.

Campbell, S.K. (2005) The infant at risk for developmental disability. In Campbell, S.K. (Ed.) Decision Making in Pediatric Neurologic Physical Therapy. Philadelphia PA: Churchill Livingstone. 
Dougherty, D. \& Luther, M. (2008) Birth to breast - A feeding care map for the NICU: Helping the extremely low birth weight infant navigate the course. Neonatal Network, 27371 377.

Elizondo, A. (1995) Nursing care management in the neonatal intensive care unit. Part 2: Developing critical pathways. Neonatal Network, 14, 11-19.

Gretebeck, R.J., Shaffer, D., \& Bishop-Kurylo, D. (1998) Clinical pathways for family-oriented developmental care in the intensive care nursery. J Perinat Neonat Nurs. 12(1), 70-80.

Jones, M.L.H. \& Smyth, K.A. (1999) Outcomes for high-risk neonates in a managed care clinical system. Nursing Case Management, 4, 71-76.

Krebs , T.L. (1998) Clinical pathway for enhanced parent and preterm infant interaction through parent education. J Perinat Neonat Nurs ,12(2), 38-49.

Mills, M.M., Sims, D.C., \& Jacob, J. (2006) Implementation and case-study results of potentially better practices to improve the discharge process in the Neonatal Intensive Care Unit. Pediatrics, 118, S124-S133.

Nelson, E.C. \& Edwards, W.H. (2009) Measure what matters. In Horbar, J.D., Leahy, K., Handyside, J. (Eds.) NICQ 2007: Improvement in Action. Burlington VT: Vermont Oxford Network (first published online at www.vtoxford.org).

Renholm, M, Leino-Kilip, H., \& Suominen, T. (2002) Critical pathways: A systematic review. J Nurs Adm, 32, 196-202.

Schwoebel, A. \& Jones, M.L.H. (1999) A clinical pathway system for the neonatal intensive care nursery. J Perinatal \& Neonatal Nurs, 13, 60-69. 
Sweeney, J.K., Heriza, C.B., \& Blanchard, Y. (2009) Neonatal physical therapy. Part I: Clinical competencies and neonatal intensive care unit clinical training models. Pediatric Physical Therapy, 21, 296-307.

Sweeney, J.K., Heriza, C.B., Blanchard, Y., \& Dusing, S. (2010) Neonatal Physical Therapy. Part II: practice frameworks and evidence-based practice guidelines. Pediatric Physical Therapy, 22, 2-16. 